Published in final edited form as:

Nat Chem. 2020 February ; 12(2): 173-179. doi:10.1038/s41557-019-0407-6.

\title{
Merging Chemoenzymatic and Radical-Based Retrosynthetic Logic For Rapid and Modular Synthesis of Oxidised Meroterpenoids
}

\author{
Jian $\mathrm{Li}^{+}$, Fuzhuo $\mathrm{Li}^{+}$, Emma King-Smith ${ }^{+}$, Hans Renata \\ Department of Chemistry, The Scripps Research Institute, 130 Scripps Way, Jupiter, FL 33458
}

\begin{abstract}
Meroterpenoids are natural products of hybrid biosynthetic origins - derived from both terpenoid and polyketide pathways - with a wealth of biological activities. Given their therapeutic potential, a general strategy to access these natural products in a concise and divergent fashion is highly desirable. Here, we report a modular synthesis of a suite of oxidized meroterpenoids using a hybrid synthetic strategy that is designed to harness the power of both biocatalytic and radicalbased retrosynthetic logic. This strategy enables direct introduction of key hydroxyl groups and rapid construction of key bonds and stereocenters, facilitating the development of a concise route (7-12 steps from commercial materials) to eight oxidized meroterpenoids from two common molecular scaffolds. This work lays the foundation for rapid access to a wide range of oxidized meroterpenoids through the use of similar hybrid strategy that combines two synthetic approaches.
\end{abstract}

Multistep chemical synthesis relies almost exclusively on the use of retrosynthetic analysis. ${ }^{1}$ Using this conceptual framework, a target molecule is disconnected through a series of reverse reactions to arrive finally at greatly simplified or commercial starting materials. As each disconnection needs to be sensible in the forward synthetic direction, the choice of bond(s) to disconnect is highly dependent on the contemporary synthetic transformations available at the practitioner's disposal. Throughout the history of organic chemistry, polar disconnections, due to their perceived robustness, permeate much of the discourse in the field. More recently, the emergence of new technologies in chemical synthesis has led to the formulation of alternative retrosynthetic strategies. Biocatalytic retrosynthesis ${ }^{2}$ has flourished into a highly powerful principle for multistep syntheses due to the unparalleled selectivity of enzymatic transformations and the ever-growing tools of protein engineering and directed evolution. ${ }^{3}$ Similarly, radical-based retrosynthetic disconnections ${ }^{4,5}$ have become increasingly popular, owing to the unique chemoselectivity and chemofidelity ${ }^{6}$ of

\footnotetext{
Users may view, print, copy, and download text and data-mine the content in such documents, for the purposes of academic research, subject always to the full Conditions of use:http://www.nature.com/authors/editorial_policies/license.html\#terms

Materials and Correspondence: Correspondence and requests for materials should be addressed to hrenata@ scripps.edu.

${ }^{+}$J.L., F.L., E.K.-S. contributed equally to this work.

Author Contributions

J.L., F.L., E.K.-S., and H.R. conceived the work. E.K.-S., J.L., and H.R. designed and conducted the initial screening of P450BM3 variants; F.L., and E.K.-S. performed the experiments described in Fig. 3; J.L. performed the experiments described in Fig. 4. H.R. wrote the manuscript; J.L., F.L., and E.K.-S. assisted in writing and editing the manuscript.

Competing Interests

The authors declare no competing interests.
} 
radicals. Despite their respective advantages, these emerging strategies have largely developed as independent entities with minimal crosstalk. Here, we describe the development of a powerful strategy to streamline access to meroterpenoid natural products (eight total) by combining the unparalleled site-selectivity of enzymatic hydroxylation with the unique reactivity profile of radical-based transformations. The efficiency of our route highlights the benefits of employing such a hybrid synthetic strategy in the synthesis of complex molecules.

Commonly isolated from fungi, meroterpenoids are a large family of hybrid terpene natural products possessing a wide range of structural diversity (Fig. 1A). ${ }^{7}$ The a-pyrone meroterpenoids (e.g., 1-5) constitute a prominent subset of this family and possess a common $\mathrm{C} 3$-oxidised drimane unit that is attached to various polyketide-derived pyrone fragments at $\mathrm{C} 11 .{ }^{8}$ Members of this family exhibit a broad range of bioactivity ranging from anti-cholinesterase activity to acyl-CoA/cholesterol acyltransferase (ACAT) inhibition. While not as common, meroterpenoids bearing a diterpene unit have also been found in nature. ${ }^{9}$ Members of this subset (e.g., 6-11) typically share a common C3-oxidised entisocopalane fragment that occurs in combination with various aromatics and have been known to exhibit anti-mycobacterial, insecticidal, and cytotoxic properties. Notably, the C3oxidised ent-isocopalane fragment is also highly prevalent in many higher-order terpenes. Despite their structural diversity, most C3-oxidised meroterpenoids share a common biosynthetic logic ${ }^{10}$ : they arise in nature from the union of polyisoprene pyrophosphate with a polyketide-derived aromatic unit, followed by enantioselective epoxidation and polyene cyclisation cascade.

Two general synthetic approaches have been developed to stereoselectively access C3oxidised meroterpenoids. The first aims to recapitulate the biosynthetic logic through the use of enantioselective dihydroxylation ${ }^{11}$ on a polyisoprenyl unit (e.g., farnesyl or geranylgeranyl), followed by epoxide formation, and Lewis acid-catalysed polyene cyclisation. This approach is linear in nature, and is often beset by slow rates of dihydroxylation, variable levels of regio- and enantioselectivity, and varying degrees of success in the biomimetic cyclisation step. For example, asymmetric dihydroxylation of farnesol derivatives is typically complicated by unwanted reaction at the internal double bond, ${ }^{12}$ and polyene cyclisation towards taondiol was reported to afford only $2 \%$ yield of the desired product. ${ }^{13}$ The dearth of efficient methods for introducing additional oxygenation on the polyisoprenyl unit further hinders the synthetic versatility of this approach for accessing more oxidised meroterpenoids. An alternative strategy commencing from Wieland-Miescher ketone has also been developed. However, this approach is strategically inefficient as it requires multiple tailoring steps on the decalin skeleton and nontrivial $\mathrm{C}-\mathrm{C}$ bond formation steps for subsequent ring construction. ${ }^{14}$ For example, the use of organolithium addition to link the terpene and arene fragments of stypodiol resulted in a diastereomeric mixture of products, ${ }^{15}$ and the use of Robinson annulation to form the tricyclic core of the decaturins was plagued by unexpected racemization. ${ }^{16}$ These shortcomings provide a strong incentive for the development of an alternative synthetic strategy.

We envisioned a synthetic approach that is based on a site-selective oxidation of a simple drimane or isocopalane-like framework (Fig. 1B). Such unit is readily available in the form 
of sclareolide (12) and sclareol (13), common feedstocks in the perfume industry that can be accrued in kilogram quantities. With respect to a-pyrone meroterpenoids, a C3-selective hydroxylation of sclareolide will furnish the minimally-oxidised A/B ring of 1-5. In turn, the lactone moiety could be readily manipulated to provide advanced intermediate $\mathbf{1 4}$ that would allow maximal divergency to access a wide range of a-pyrone meroterpenoids. A similar strategy for the synthesis of quinone sesquiterpenoids has previously been conceived through the intermediacy of boronosclareolide. ${ }^{17}$ However, this approach suffers from the lack of ability to introduce additional oxygenations at $\mathrm{C} 3, \mathrm{C} 9$, and $\mathrm{C} 11$. To address these limitations, we envisioned the conversion of the aldehyde moiety of $\mathbf{1 4}$, to the corresponding olefin, which would offer versatile installation ${ }^{18}$ of various functionalities at $\mathrm{C} 9$ and $\mathrm{C} 11$. While this transform would lead to a retron containing multiple olefins, we reasoned that the abundance of options for olefin functionalization would allow for the identification of a suitable method with the desired chemoselectivity.

Similarly, a C3-selective hydroxylation of sclareol will generate a synthetic intermediate that not only possesses the correct oxidation state at $\mathrm{C} 3$, but also the appropriate functional handles for the construction of the remaining rings of $6-11$. In the forward sense, a $\mathrm{C}-\mathrm{C}$ bond formation event would afford advanced intermediate $\mathbf{1 5}$, which acts as a divergency point to access a wide range of diterpenic meroterpenoids. It is worth noting that related tricyclic motifs have previously been constructed from $\mathbf{1 3}$. However, work in this area has been dominated by two electron-based transformations. For example, the only total synthesis of makassaric acid proceeded from $\mathbf{1 3}$ and enlisted the use of organolithium addition to an aldehyde, followed by Barton deoxygenation. ${ }^{19}$ Adaptation of such transformation onto routes that feature 3-hydroxysclareol would necessitate undesired protecting group manipulations and functional group interconversions. In contrast, we believe that radicalbased transformations, with their unique reactivity profile, would allow us to bypass such concession steps.

From a medicinal chemistry perspective, this overall strategy provides an ideal synthetic blueprint to target a wide spectrum of meroterpenoids from just two common intermediates. Nevertheless, the realization of this strategy is not without its challenges. Numerous chemical methods have been reported for selective $\mathrm{C}-\mathrm{H}$ functionalisation of $\mathbf{1 2} .^{20,21,22,23}$ However, they rely on the innate reactivity of the carbocyclic scaffold and result predominantly in $\mathrm{C} 2$ modification. While whole-cell fungal biotransformation of $\mathbf{1 2}$ has been reported to give C3-oxidised product, it is typically accompanied by over-oxidation to the ketone product and oxidation at other positions. ${ }^{24}$ At the outset of our work, C3-selective enzymatic hydroxylation of $\mathbf{1 2}$ has been reported to take place with a $\mathrm{P} 450_{\mathrm{BM} 3}$ variant ${ }^{25} \mathrm{II}-$ H8 (15 mutations from wild type), or CYP101B1, a P450 monooxygenase from $N$. aromaticivorans DSM12444. ${ }^{26}$ However, these bioconversions have only been carried out on up to $50 \mathrm{mg}$ scale under high dilution conditions $(1 \mathrm{mM}$ substrate concentration with $c$. 1000 total turnover number (TTN)), and their scalability remains to be addressed. Additionally, oxidation of sclareol has only been attempted with whole-cell fungal biotransformation and was reported to result in non-selective oxidation. ${ }^{24,27}$ 


\section{Results and discussion}

We began by conducting a brief survey of several $\mathrm{P}^{4} 50_{\mathrm{BM} 3}$ variants harbouring similar mutations to II-H8 for the hydroxylation of sclareolide and sclareol (Fig. 2). With an eye towards practical and inexpensive hydroxylation method, small-scale screening of these variants was performed with lysate of $E$. coli cells expressing both the $\mathrm{P} 450_{\mathrm{BM} 3}$ variant and a thermostabilised phosphate dehydrogenase (Opt13) for NADPH recycling. ${ }^{28}$ Here, we chose to employ pET22b(+)- and pRSF-based vectors for overexpression of the $\mathrm{P}_{450} 0_{\mathrm{BM}}$ variant and Opt13 respectively, as they possess compatible origins of replication and allow the desired genes to be expressed under the control of strong T7lac promoter. Alanine scanning ${ }^{29}$ on variant 1857 (four amino acid substitutions from II-H8) revealed several mutants with varying levels of hydroxylation activity on 12. Gratifyingly, variant 1857 V328A (BM3 MERO1) afforded $>95 \%$ conversion to 3- $(\mathrm{OH})$-sclareolide (corresponding to ca. $5000 \mathrm{TTN}$ ), with no detectable presence of the undesired C2-hydroxylated product. Mutations T235A and R471A, previously reported to enhance the organic solvent tolerance of $\mathrm{P}_{450} 0_{\mathrm{BM} 3},{ }^{30}$ were introduced in an attempt to perform the biocatalytic oxidation at higher substrate concentration and amount of organic cosolvent. Unfortunately, this variant (BM3 MERO2) proved to be inferior to BM3 MERO1 for the hydroxylation of $\mathbf{1 2}$. Reversion of thermostabilising mutations $\mathrm{C} 47 \mathrm{R}$ and $194 \mathrm{~K}^{29}$ resulted in variant BM3 MERO3, which showed comparable conversion to BM3 MERO1 on both small-scale and preparative-scale reactions, suggesting that the thermostabilising mutations are not necessary for achieving high hydroxylation activity on 12. The aforementioned variants were also tested for their ability to hydroxylate sclareol, revealing several variants with modest hydroxylation activity (ca. 30\% conversion). Importantly, all variants examined proved selective for C3 hydroxylation and provided initial validation for our hybrid strategy in the synthesis of diterpenic meroterpenoids.

With optimized $\mathrm{P} 450_{\mathrm{BM}}$ variants for sclareolide hydroxylation in hand, we sought to establish a robust route to prepare a suitable precursor for fragment coupling with the pyrone unit (Fig. 3A). Enzymatic hydroxylation of sclareolide could be routinely conducted on gram scale with BM3 MERO1 with excellent conversion and isolated yield. The supplementation of Opt13 allowed for substoichiometric use of NADPH, lowering the overall cost of conducting large-scale reactions. As a testament to the scalability of the biotransformation, lactone $\mathbf{1 8}$ has been prepared in $>4 \mathrm{~g}$ quantity to date. Intermediate 14 could be quickly accessed through tailoring of the C-ring lactone in 2 steps from 18, setting the stage for the key coupling with pyrones 19-21. In contrast, preparation of 14 from Wieland-Miescher ketone is projected to require at least 8 steps, highlighting the tactical directness of our strategy. A brief survey of Brønsted and Lewis acids yielded phosphoric acid $\mathbf{2 2}^{31}$ as an ideal catalyst for in situ alcohol dehydration and formal [3+3] union. Under these conditions, $\mathbf{2 3}$ and $\mathbf{2 4}$ were obtained in 55\% and 64\% yields, respectively. Coupling with pyrone $\mathbf{2 1}$ necessitated the use of enal $\mathbf{2 5}$ as a substrate, which readily underwent a formal [ $3+3$ ] with $\mathbf{2 1}$ in the presence of piperidinium acetate. ${ }^{32}$

With the carbon framework fully installed, attention turned to the reduction of the C9-C11 alkene. A gamut of hydrogenation conditions in the presence of $\mathrm{Rh}, \mathrm{Pt}$, and $\mathrm{Pd}$ catalysts was surveyed, only to yield reduction and/or over-reduction of the pyrone motif. We reasoned 
that hydrogen atom transfer-based (HAT-based) hydrogenation would provide a viable solution to this problem. ${ }^{33,34}$ The steric bulk of the catalyst would suppress any undesired reactivity with the tetrasubstituted alkene, and between the two trisubstituted alkenes, reduction of $\Delta^{9,11}$ should be preferred due to the greater stability of the incipient radical at C9. Furthermore, HAT-based reduction is known to deliver the thermodynamic hydrogenation product, providing trans-decalin selectively from $\Delta^{9,10}$-octalin. Indeed, subjecting 23, 24, and 26 to Shenvi's ${ }^{33}$ reduction conditions $\left(\mathrm{Mn}(\mathrm{dpm})_{3}\right.$, tert-butyl hydrogen peroxide (TBHP), and $\mathrm{PhSiH}_{3}$ ) provided arisugacin $\mathrm{F}(\mathbf{1})$, phenylpyropene $\mathrm{C}(\mathbf{2})$, and pyripyropene $\mathrm{E}(4)$ in moderate to high yields and excellent diastereoselectivities (Fig. 3B, $3 \mathrm{C})$.

The modularity of the designed sequence was further underscored by the ability to obtain phenylpyropene $F(3)$, a more oxidised member of the a-pyrone meroterpenoid family, in a concise manner (Fig. 3D). Here, aldehyde 14 was converted to acid chloride 27, which was then subjected to a Friedel-Crafts acylation with pyrone 20, followed by a cyclisation/ reduction sequence to complete the synthesis of $\mathbf{3}$ in 11 steps. The C9-C11 alkene proved to be a versatile functional handle, as Mukaiyama hydration ${ }^{35}$ on this moiety proceeded in a highly selective fashion (>20:1 dr) to generate the corresponding $3^{\circ}$ alcohol at C9 (Fig. 3E). In contrast, previous approach to generate this alcohol required the intermediacy of the corresponding epoxide, which was then subjected to nucleophilic opening and alcohol reduction. In combination with $\mathrm{C} 3$ alcohol oxidation, this approach enabled rapid access to 5-deoxyterreulactone C (28) from $\mathbf{2 3}$ in 64\% yield over 2 steps.

In parallel, route scouting for efficient access to diterpenic meroterpenoids was undertaken, affording a five-step synthesis of tricycle $\mathbf{3 1}$ as a single diastereomer (Fig. 4A). Key to this success is the use of HAT-based intramolecular Giese coupling ${ }^{36}$ to forge the $\mathrm{C}$ ring with complete diastereoselectivity and excellent yield. This outcome could be rationalised by invoking rapid pyramidalisation of the incipient $3^{\circ}$ radical and cyclic stereocontrol imposed by the rigid trans-decalin and butyrolactone moieties. In contrast, previous approach to construct methyl ent-isocopalate from sclareol using polar 2-electron-based transformations resulted only in $82 \%$ de of the desired isomer. ${ }^{37}$ Intermediates 13, 29-31 were tested for enzymatic hydroxylation with various $\mathrm{P} 450_{\mathrm{BM} 3}$ mutants. From this combinatorial experiment, the pairing of acid $\mathbf{3 1}$ and $\mathrm{P} 450_{\mathrm{BM} 3}$ variant $\mathrm{BM} 3 \mathrm{MERO} 1$ yielded the most promising outcome (33\% yield). Further alanine scanning on MERO1 identified variant MERO1 L75A as a superior biocatalyst, providing the desired C3-oxidized product $\mathbf{3 2}$ in $62 \%$ yield with complete regio- and diastereoselectivity. Gratifyingly, this key transformation could be routinely conducted on gram-scale without any appreciable decrease in conversion and isolated yield.

At this stage, radical cross-coupling employing redox-active ester derivatives ${ }^{38}$ of $\mathbf{3 2}$ was attempted to access taondiol and chevalone A. However, in both cases, no desired product formation was observed. As a workaround, $\mathbf{3 2}$ was first converted to the corresponding iodide (34), which was then subjected to nickel-catalysed cross coupling using Weix's procedure ${ }^{39}$ with $\mathbf{3 6}$ and $\mathbf{3 7}$ (Fig. 4B). Using this procedure, the desired coupling products could be obtained in $85 \%$ and $76 \%$ yields, respectively. Finally, acid-catalysed cyclisations forged the final dihydropyran rings and completed the total syntheses of $\mathbf{1 0}$ and $\mathbf{1 1}$. The 
versatility of this synthetic approach is further highlighted by the ability to use the same iodo intermediate 34 to access decaturin E (6) and stypodiol (8) in a modular and efficient manner (Fig. 4C). Treatment of $\mathbf{3 4}$ with $t \mathrm{BuOK}$ afforded the corresponding diene (35) and set the stage for the final fragment coupling steps. The synthesis of $\mathbf{6}$ was realised by the use of a single electron transfer-based (SET-based) formal [3 +2] coupling of $\mathbf{3 5}$ and pyrone $\mathbf{2 1}$. Initial attempts to realise this transformation in the presence of ceric ammonium nitrate ${ }^{40}$ were accompanied by competing oxidation of the $\mathrm{C} 3$ alcohol to the ketone. We hypothesised that increasing the equivalents of pyrone $\mathbf{2 1}$ would allow preferential single electron oxidation of this fragment and suppress undesired alcohol oxidation. Indeed, increasing the equivalents of pyrone 21 to 5 equivalents led to formation of $\mathbf{6}$ in $83 \%$ yield with no observable over-oxidation side product. Attempts to effect this CAN-mediated coupling with phenol $\mathbf{3 9}$ led to exclusive oxidation of $\mathbf{3 9}$ to the corresponding quinone. Hypothesising that electrochemical methods ${ }^{41}$ would allow a more controlled electron delivery to the phenol fragment, we subjected $\mathbf{3 5}$ and $\mathbf{3 9}$ to constant potential electrolysis. Gratifyingly, this approach was able to provide the desired [ $3+2]$ coupling product $\mathbf{4 0}$ as a single diastereomer in $62 \%$ yield. At this stage, the $\mathrm{C} 12-\mathrm{C} 13$ olefin needed to be reduced to the thermodynamic product containing an equatorial methyl group at C13. Given its thermodynamic preference, we turned to Shenvi's HAT hydrogenation, which provided the correct stereochemical disposition at C13 with 10:1 dr. Once again, these radical-based conditions were found to be crucial as other reduction conditions led predominantly to the formation of the wrong diastereomer. Routine phenol demethylation finally completed the total synthesis of $\mathbf{8}$. Stypodiol has been shown to undergo ready conversion to stypodione ${ }^{42}$ and stypotriol. ${ }^{43}$ Thus, our approach also constitutes a 13-step and 14-step formal synthesis of stypodione and stypotriol, respectively.

The collective synthesis of various meroterpenoids presented in this work proceeded in 7-12 steps, comprising some of the most concise routes to the target molecules to date. Our hybrid approach results not only in favourable step count, but also significant improvement in overall yields for most of the targets (up to five-fold improvement, see Supplementary Table 1 for comparison). This work also constitutes the first total syntheses of $\mathbf{2 , 3 , 6}$ and $\mathbf{1 1}$. The orthogonal site-selectivity ${ }^{44,45,46}$ of biocatalytic $\mathrm{C}-\mathrm{H}$ oxidation methods provided the opportunity to develop a novel disconnection for terpene synthesis and its combination with radical-based transformations $s^{4,5}$ allowed us to achieve exquisite stereocontrol and chemoselectivity in all of the key bond forming steps. Such features represent a distinct departure from previous approaches to bioactive meroterpenoids and highlight the benefits of combining $45,47,48$ modern synthetic paradigms in complex molecule synthesis. Importantly, eight synthetic targets could be accessed divergently from just two key intermediates, suggesting that this route can be readily adapted for the preparation of further synthetic derivatives as well as other meroterpenoid natural products bearing similar decalin architecture. ${ }^{49,50}$ Work in this area is underway and will be reported in due course. 


\section{Data Availability}

Full experimental details, information about enzyme variants, and Supplementary Tables 19 are available in the Supplementary Information. Any additional information is available from the corresponding author upon request.

\section{Supplementary Material}

Refer to Web version on PubMed Central for supplementary material.

\section{Acknowledgements}

This work is supported by the National Institutes of Health Grant GM128895. We thank Profs. Phil S. Baran and Keary M. Engle for discussions and assistance in manuscript preparation. We acknowledge Prof. Frances H. Arnold (California Institute of Technology) and Prof. Huimin Zhao for providing plasmids encoding for P450 BM3 variant 1857 and phosphite dehydrogenase variant Opt13, respectively. We thank the Shen lab and the Roush lab for generous access to their instrumentations.

\section{References}

1. Corey EJ \& Cheng X-M The Logic of Chemical Synthesis (Wiley, 1995).

2. Turner NJ \& O’Reilly E Biocatalytic retrosynthesis. Nat. Chem. Biol. 9, 285-288 (2013). [PubMed: 23594772]

3. Turner NJ Directed evolution drives the next generation of biocatalysts. Nat. Chem. Biol. 5, 567573 (2009). [PubMed: 19620998]

4. Hung K, Hu X \& Maimone T Total synthesis of complex terpenoids employing radical cascade processes. Nat. Prod. Rep. 35, 174-202 (2018). [PubMed: 29417970]

5. Smith JM, Harwood SJ \& Baran PS Radical retrosynthesis. Acc. Chem. Res. 51, 1807-1817 (2018). [PubMed: 30070821]

6. Green SA et al. The high chemofidelity of metal-catalyzed hydrogen atom transfer. Acc. Chem. Res. 51, 2628-2640 (2018). [PubMed: 30406655]

7. Matsuda Y \& Abe I Biosynthesis of fungal meroterpenoids. Nat. Prod. Rep. 33, 26-53 (2016). [PubMed: 26497360]

8. Sunazuka T \& Ōmura S Total synthesis of a-pyrone meroterpenoids, novel bioactive microbial metabolites. Chem. Rev. 105, 4559-4580 (2005). [PubMed: 16351054]

9. Macías FA, Carrera C \& Galindo JCG Brevianes revisited. Chem. Rev. 114, 2717-2732 (2014). [PubMed: 24359217]

10. Itoh $\mathrm{T}$ et al. Reconstitution of a fungal meroterpenoid biosynthesis reveals the involvement of a novel family of terpene cyclases. Nat. Chem. 2, 858-864 (2010). [PubMed: 20861902]

11. Corey EJ, Noe MC \& Lin S A mechanistically designed bis-cinchona alkaloid ligand allows position- and enantioselective dihydroxylation of farnesol and other oligoprenyl derivatives at the terminal isopropylidene unit. Tetrahedron Lett. 36, 8741-8744 (1995).

12. Smith AB III,, Kinso T, Sunazuka T \& Ōmura S. Biomimetic total synthesis of the ACAT inhibitor (+)-pyripyropene E. Tetrahedron Lett. 37, 6461-6464 (1996).

13. Kumanireng AS, Kato T \& Kitahara Y Cyclization of polyenes x. biogenetic type synthesis of $d l-$ taodiol. Chem. Lett. 2, 1045-1047 (1973).

14. Nagamitsu $\mathrm{T}$ et al. Total synthesis of (+)-pyripyropene A, a potent, orally bioavailable inhibitor of Acyl-CoA:cholesterol acyltransferase. J. Org. Chem. 60, 8126-8127 (1995).

15. Abad A et al. An efficient stereoselective synthesis of stypodiol and epistypodiol. J. Org. Chem. 63, 5100-5106 (1998).

16. Takikawa H, Imamura Y \& Sasaki M Synthesis and absolute configuration of brevione $B$, an allelochemical isolated from Penicillium sp.. Tetrahedron 62, 39-48 (2006). 
17. Dixon DD, Lockner JW, Zhou Q \& Baran PS Scalable, divergent synthesis of meroterpenoids via "borono-sclareolide". J. Am. Chem. Soc. 134, 8432-8435 (2012). [PubMed: 22583115]

18. Crossley SWM, Obradors C, Martinez RM \& Shenvi RA Mn-, Fe-, and Co-catalyzed radical hydrofunctionalizations of olefins. Chem. Rev. 116, 8912-9000 (2016). [PubMed: 27461578]

19. Basabe $P$ et al. Synthesis of (+)-makassaric acid, a protein kinase MK2 inhibitor. Tetrahedron 66, 6008-6012 (2010).

20. Chen MS \& White MC Combined effects on selectivity in Fe-catalyzed methylene oxidation. Science 327, 566-571 (2010). [PubMed: 20110502]

21. Quinn RK et al. Site-selective aliphatic C-H chlorination using N-chloroamides enables a synthesis of chlorolissoclimide. J. Am. Chem. Soc. 138, 696-702 (2016). [PubMed: 26694767]

22. Kawamata, et al. Scalable, electrochemical oxidation of unactivated C-H bonds. J. Am. Chem. Soc. 139, 7448-7451 (2017). [PubMed: 28510449]

23. Chiappini ND, Mack JBC \& Du Bois J Intermolecular C(sp3)-H amination of complex molecules. Angew. Chem. Int. Ed. 57, 4956-4959 (2018).

24. Frija LMT, Frade RFM \& Afonso CAM Isolation, chemical, and biotransformation routes of labdane-type diterpenes. Chem. Rev. 111, 4418-4452 (2011). [PubMed: 21618966]

25. Zhang K, El Damaty S \& Fasan R P450 fingerprinting method for rapid discovery of terpene hydroxylating P450 catalysts with diversified regioselectivity. J. Am. Chem. Soc. 133, 3242-3245 (2011). [PubMed: 21341707]

26. Hall EA, Sarkar MR, Lee JHZ, Munday SD \& Bell SG Improving the monooxygenase activity and the regio- and stereoselectivity of terpenoid hydroxylation using Ester directing groups. ACS Catal. 6, 6306-6317 (2016).

27. Aranda $\mathrm{G}$ et al. Microbial transformation of diterpenes: Hydroxylation of sclareol, manool and derivatives by Mucor plumbeus. Tetrahedron 47, 8339-8350 (1991).

28. McLachlan MJ, Johannes TW \& Zhao H Further improvement of phosphite dehydrogenase thermostability by saturation mutagenesis. Biotechnol. Bioeng. 99, 268-274 (2008). [PubMed: 17615560]

29. Lewis JC et al. Combinatorial alanine substitution enables rapid optimization of cytochrome $\mathrm{P}_{50} 0_{\mathrm{BM} 3}$ for selective hydroxylation of large substrates. ChemBioChem 11, 2502-2505 (2010). [PubMed: 21108271]

30. Wong TS, Arnold FH \& Schwaneberg U Laboratory evolution of cytochrome P450 BM-3 monooxygenase for oganic co-solvents. Biotechnol. Bioeng. 85, 351-358 (2004). [PubMed: 14748091]

31. Hubert $\mathrm{C}$ et al. Brønsted acid-catalyzed synthesis of pyrans via a formal [3+3] cycloaddition. Adv. Synth. Catal. 350, 40-42 (2008).

32. Hsung RP, Kurdyumov AV \& Sydorenko N A formal [3 + 3] cycloaddition approach to naturalproduct synthesis. Eur. J. Org. Chem. 1, 23-44 (2005).

33. Iwasaki K, Wan KK, Oppedisano A, Crossley SWM \& Shenvi RA Simple, chemoselective hydrogenation with thermodynamic stereocontrol. J. Am. Chem. Soc. 136, 1300-1303 (2014). [PubMed: 24428640]

34. King SM, Ma X \& Herzon SB A method for the selective hydrogenation of alkenyl halides to alkyl halides. J. Am. Chem. Soc. 136, 6884-6887 (2014). [PubMed: 24824195]

35. Isayama $S \&$ Mukaiyama T A new method for preparation of alcohols from olefins with molecular oxygen and phenylsilane by the use of bis(acetylacetonato)cobalt(II). Chem. Lett. 18, 1071-1074 (1989).

36. Lo JC et al. Fe-catalyzed C-C bond construction from olefins via radicals. J. Am. Chem. Soc. 139, 2484-2503 (2017). [PubMed: 28094980]

37. Hua S-K, Wang J, Chen X-B, Xu Z-Y \& Zeng B-B Scalable synthesis of methyl ent-isocopalate and its derivatives. Tetrahedron 67, 1142-1144 (2011).

38. Sandfort F, O’Neill MJ, Cornella J, Wimmer L \& Baran PS Alkyl-(hetero)aryl bond formation via decarboxylative cross-coupling: a systematic analysis. Angew. Chem. Int. Ed. 56, 3319-3323 (2017). 
39. Everson DA, Shrestha R \& Weix DJ Nickel-catalyzed reductive cross-coupling of aryl halides with alkyl halides. J. Am. Chem. Soc. 132, 920-921 (2010). [PubMed: 20047282]

40. Yokoe $\mathrm{H}$ et al. Enantiocontrolled total syntheses of breviones A, B, and C. J. Am. Chem. Soc. 133, 8854-8857 (2011). [PubMed: 21557626]

41. Chiba K, Fukuda M, Kim S, Kitano Y \& Tada M Dihydrobenzofuran synthesis by an anodic [3+ 2] cycloaddition of phenols and unactivated alkenes. J. Org. Chem. 64, 7654-7656 (1999).

42. Falck JR et al. Total synthesis of the spiro-o-benzoquinonefuran (-)-stypoldione. J. Am. Chem. Soc. 115, 11606-11607 (1993).

43. Gerwick WH \& Fenical W Ichthyotoxic and cytotoxic metabolites of the tropical brown alga Stypopodium zonale (Lamouroux) papenfuss. J. Org. Chem. 46, 22-27 (1981).

44. King-Smith E, Zwick CR III \& Renata H Applications of oxygenases in the chemoenzymatic total synthesis of complex natural products. Biochemistry 57, 403-412 (2018). [PubMed: 29140086]

45. Loskot SA, Romney DK, Arnold FH \& Stoltz BM Enantioselective total synthesis of nigelladine A via late-stage $\mathrm{C}-\mathrm{H}$ oxidation enabled by an engineered P450 enzyme. J. Am. Chem. Soc. 139, 10196-10199 (2017). [PubMed: 28721734]

46. Lowell AN, et al. Chemoenzymatic total synthesis and structural diversification of tylactone-based macrolide antibiotics through late-stage polyketide assembly, tailoring, and $\mathrm{C}-\mathrm{H}$ functionalization. J. Am. Chem. Soc. 139, 7913-7920 (2017). [PubMed: 28525276]

47. Latham J, Henry J-M, Sharif HH, Menon BRK, Shepherd SA \& Micklefield J Integrated catalysis opens new arylation pathways via regiodivergent enzymatic $\mathrm{C}-\mathrm{H}$ activation. Nature Commun. 7, 11873 (2016). [PubMed: 27283121]

48. Durak JJ, Payne JT \& Lewis JC Late-stage diversification of biologically-active molecules via chemoenzymatic C-H functionalization. ACS Catal. 6, 1451-1454 (2016). [PubMed: 27274902]

49. Kulcitki V, Harghel P \& Ungur N Unusual cyclic terpenoids with terminal pendant prenyl moieties: from occurrence to synthesis. Nat. Prod. Rep. 31, 1686-1720 (2014). [PubMed: 25118808]

50. Domingo V, Arteaga JF, Quilez del Moral JF \& Barrero AF Unusually cyclized triterpenes: occurrence, biosynthesis and chemical synthesis. Nat. Prod. Rep. 26, 115-134 (2009). [PubMed: 19374125] 
a<smiles>CC1CCC2C(C)(C)C(O)CCC2(C)C1C</smiles>

3-(OH)-drimane unit<smiles>COc1ccc(-c2cc3c(c(=O)o2)[C@H]2C[C@]4(C)CC[C@@H](O)C(C)(C)[C@]4(C)CC[C@@]2(C)O3)cc1</smiles>

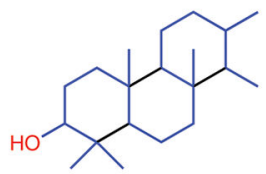

3-(OH)-ent-isocopalane unit

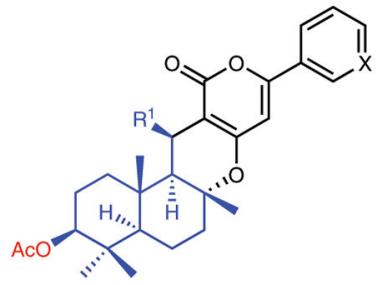

$\mathrm{X}=\mathrm{CH}, \mathrm{R}^{1}=\mathrm{H}$; phenylpyropene $\mathrm{C}$ (2)

$\mathrm{X}=\mathrm{CH}, \mathrm{R}^{1}=\mathrm{OH}$; phenylpyropene $\mathrm{F}(\mathbf{3})$

$\mathrm{X}=\mathrm{N}, \mathrm{R}^{1}=\mathrm{H}$; pyripyropene $\mathrm{E}(\mathbf{4})$

$\mathrm{X}=\mathrm{N}, \mathrm{R}^{1}=\mathrm{OH}$; pyripyropene $\mathrm{G}(\mathbf{5})$

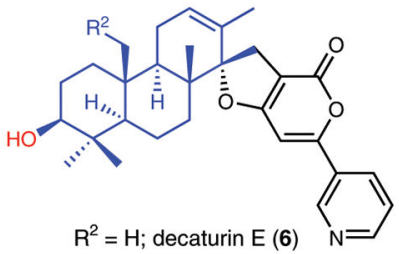

$\mathrm{R}^{2}=\mathrm{OH}$; decaturin $\mathrm{F}(\mathbf{7})$

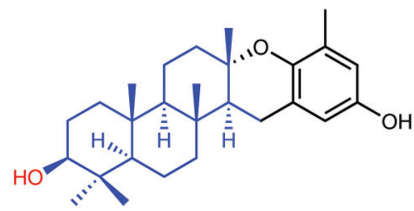

Taondiol (10)

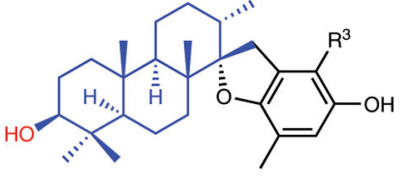

$\mathrm{R}^{3}=\mathrm{H}$; stypodiol (8)

$\mathrm{R}^{3}=\mathrm{OH}$; stypotriol (9)

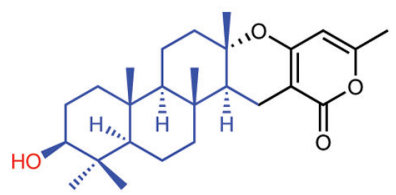

Chevalone A (11)

b

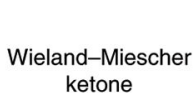<smiles>CC12CCC(=O)C=C1CCCC2=O</smiles>

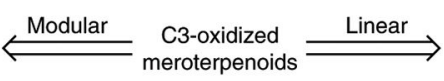

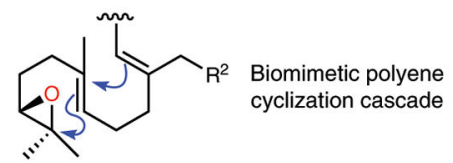

c

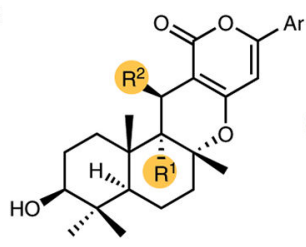

Additional oxidations at $\mathrm{C} 9$ and $\mathrm{C} 11$ possible
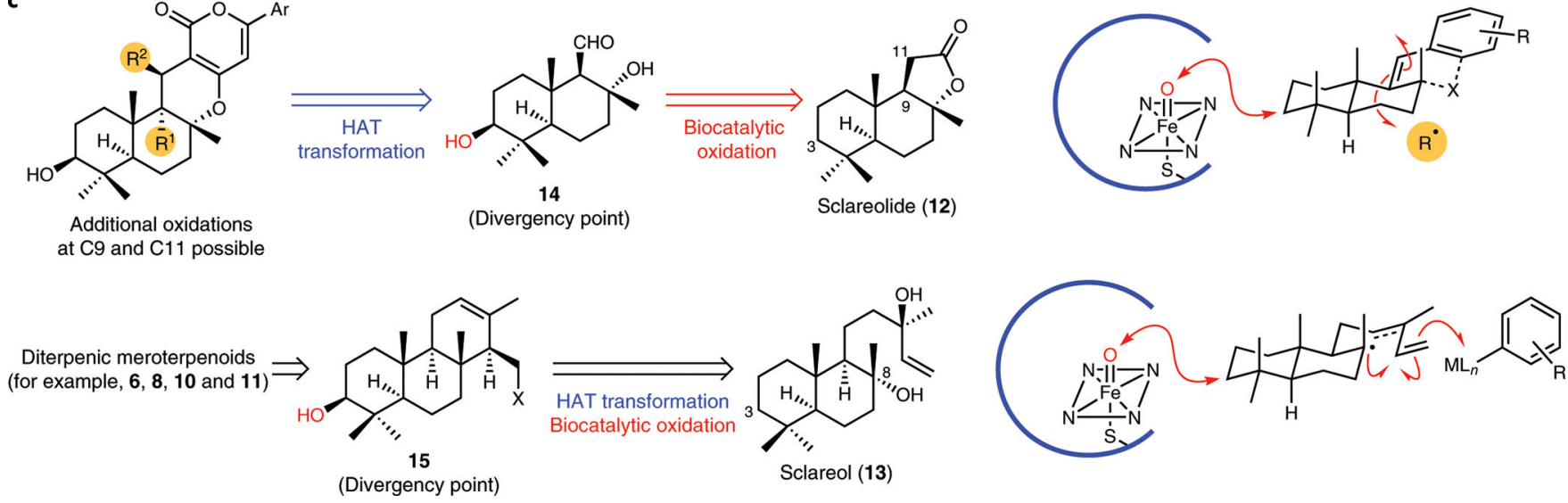

Figure 1. Combining chemoenzymatic and radical-based retrosynthetic logic for collective synthetic access to oxidised meroterpenoids.

a. C3-oxidised meroterpenoids share a common 3-(OH)-drimane or 3-(OH)-ent-isocopalane unit. These ubiquitous decalin motifs are found in over 100 terpenoids. b. Prior synthetic strategies employed to access C3-oxidised meroterpenoids typically rely on inefficient manipulation of Wieland-Miescher ketone or a biomimetic polyene cyclisation cascade. These 2 electron-based strategies are tactically indirect, involve inefficient functional group manipulations, and result in sub-optimal chemo- and diastereoselectivity. c. In our synthesis design, the a-pyrone meroterpenoids were envisioned to arise from a range of radical-based transformations to install the requisite functional groups at $\mathrm{C} 9$ and $\mathrm{C} 11$. Intermediate 14 would be prepared via biocatalytic oxidation at C3 of sclareolide (12). Conversely, the diterpenic meroterpenoids could be accessed from a common intermediate 15, which would be synthesised from sclareol (13) via biocatalytic oxidation at $\mathrm{C} 3$ and radical-based transformation(s) to forge the $\mathrm{C}$ ring, including radical-based cross coupling. Overall, the 
unique reactivity profile of radicals (chemoselectivity and chemofidelity), and siteselectivity of enzymatic oxidation will allow a more direct access to a wide range of meroterpenoids. Key disconnections for pyrone meroterpenoids (above) and diterpenic meroterpenoids (below) are shown on the right. HAT, hydrogen atom transfer. 

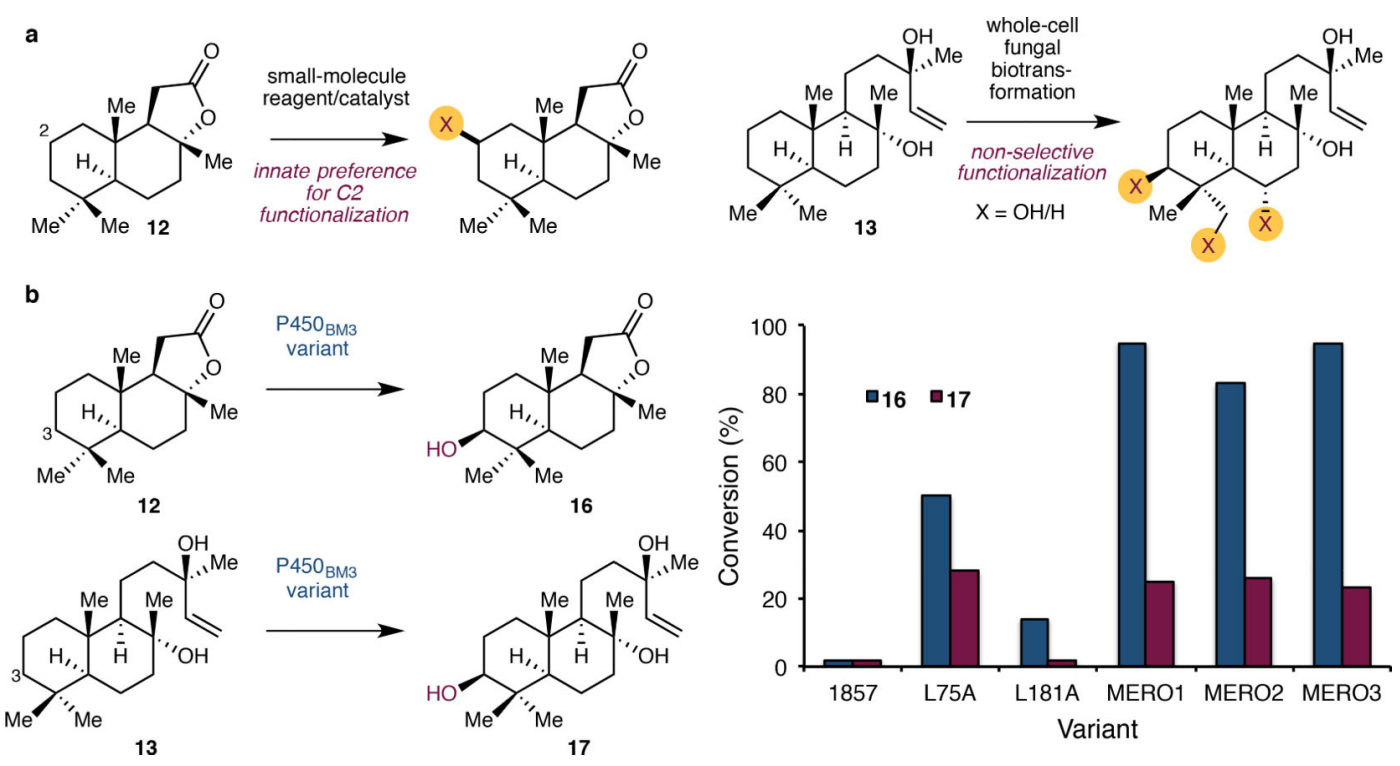

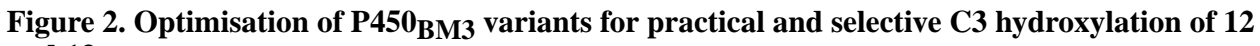
and 13.

a. Chemical methods reported for $\mathrm{C}-\mathrm{H}$ functionalisation of sclareolide (12) have an innate preference for $\mathrm{C} 2$ functionalisation ${ }^{20-23}$ while whole-cell fungal biotransformation of sclareol (13) has been reported to form regioisomeric mixture of hydroxylated products. $\mathbf{b}$. Alanine scanning on variant 1857 led to the identification of several variants with improved hydroxylation activities on 12 and 13. Among the variants tested, variants BM3 MERO1 and BM3 MERO3 were identified as the optimal variants for the hydroxylation of $\mathbf{1 2}$ and 13.

The identities of the latter variants are as follows: BM3 MERO1 = 1857 V328A, BM3 MERO2 = 1857 T235A V328A R471A, BM3 MERO3 = 1857 C47R I94K V328A. Reaction conditions for terpene hydroxylation: 12 or $13(5.0 \mathrm{mM}), \mathrm{NADP}^{+}(1.0 \mathrm{mM}), \mathrm{NaHPO}_{3}(100$ $\mathrm{mM}$ ), clarified lysate of $E$. coli BL21(DE3) expressing the appropriate $\mathrm{P}_{450}{ }_{\mathrm{BM}}$ variant and Opt13 (suspension in $50 \mathrm{mM} \mathrm{pH} 8.0 \mathrm{kPi}$, pre-lysis $\mathrm{OD}_{600}=15$ ), $20 \mathrm{~h}$ at $20^{\circ} \mathrm{C}$. 
a

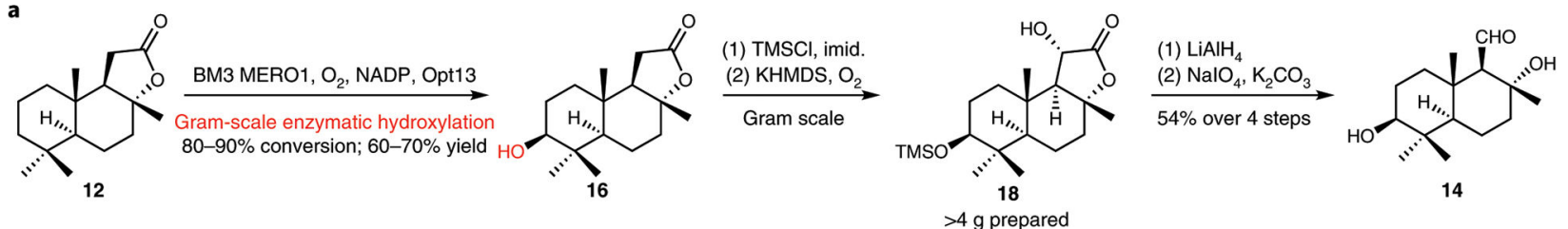

b
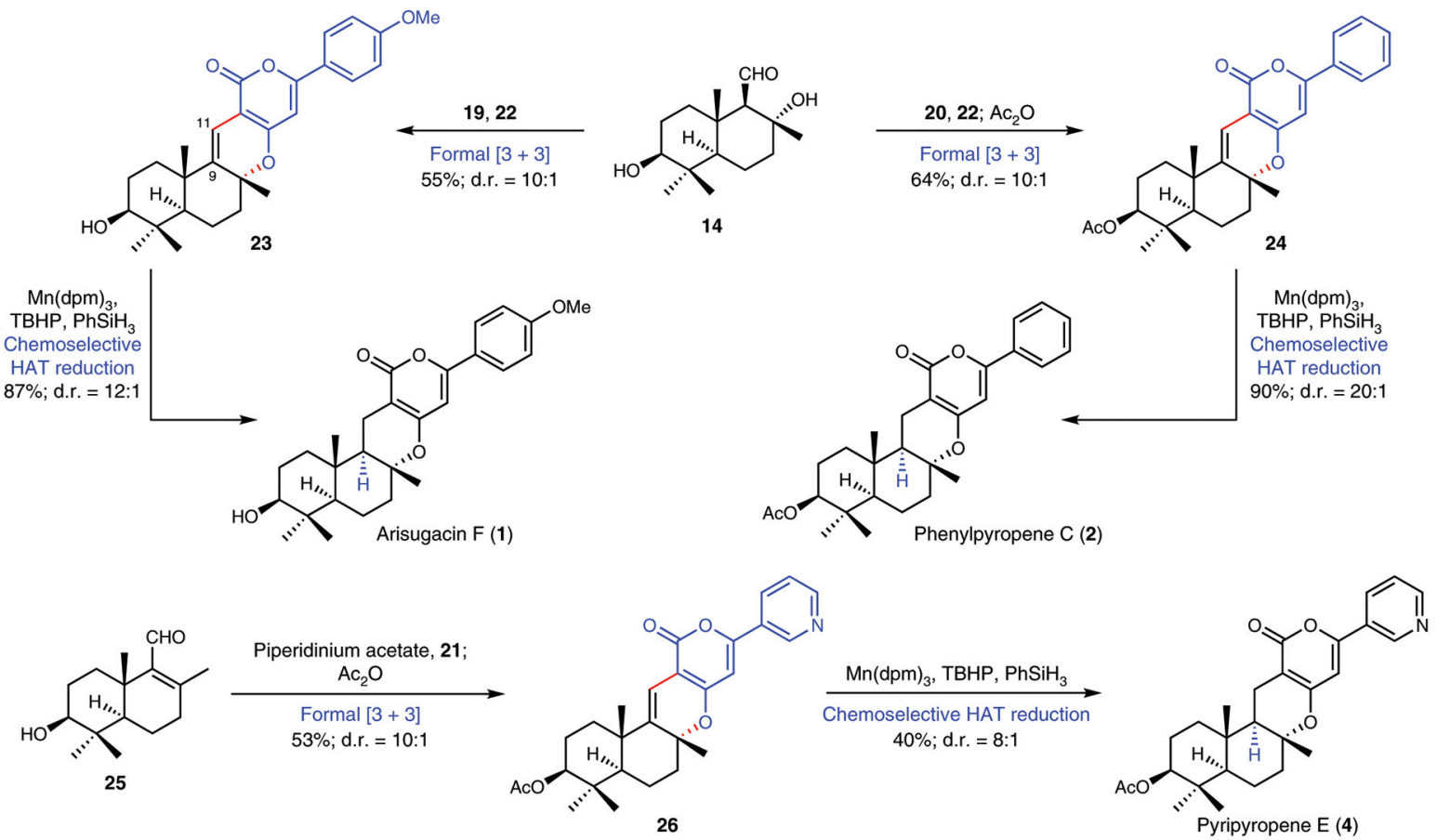

d

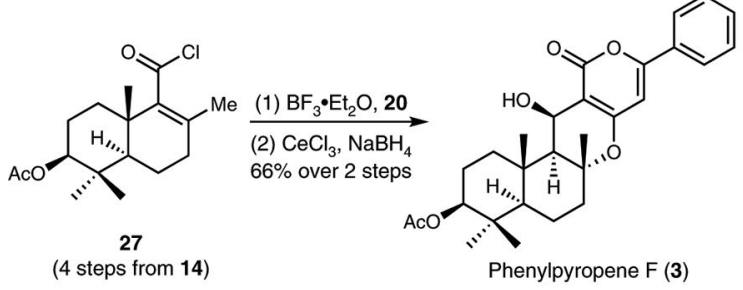

e Pyripyropene E (4)<smiles>COc1ccc(-c2cc(O)cc(=O)o2)cc1</smiles><smiles>O=c1cc(O)cc(-c2ccccc2)o1</smiles><smiles>O=c1cc(O)cc(-c2cccnc2)o1</smiles><smiles>O=P1(O)Oc2ccccc2-c2ccccc2O1</smiles>

(1) $\mathrm{Mn}(\mathrm{dpm})_{3}$

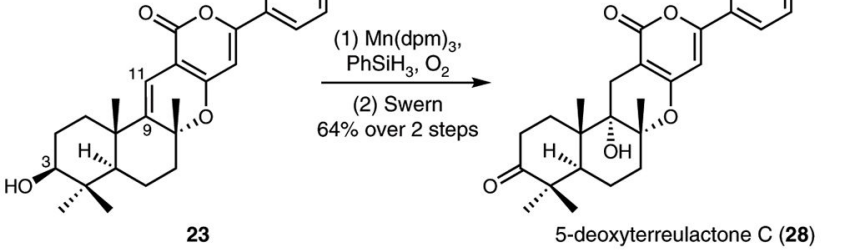

Figure 3. Modular chemoenzymatic synthesis of a-pyrone meroterpenoids.

a. Gram-scale biocatalytic hydroxylation of sclareolide (12), followed by oxidative degradation of its lactone moiety, enabled rapid access to key aldehyde 14. b. Compound 14 was converted to arisugacin $F(\mathbf{1})$ and phenylpyropene $C(2)$ via [3 3 ] coupling to append the appropriate pyrone units and HAT hydrogenation to selectively reduce the C9-C11 olefin. c. Compound 14 was dehydrated to enal 25, which in turn could be converted to pyripyropene E (4) via a similar [3 + 3] coupling/HAT hydrogenation sequence. 1, 2 and 4 are obtained in 7-8 steps from 12; biocatalytic installation of $\mathrm{C} 3-\mathrm{OH}$ and the use of radical- 
based logic for reduction at $\mathrm{C} 9$ are important transformations in their syntheses. d. Acid chloride 27, available from 14 in 4 steps, was converted to phenylpyropene $F(3)$ via [3 + 3] coupling, followed by Luche reduction of the $\mathrm{C} 11$ ketone. e. A chemoselective Mukaiyama hydration of the C9-C11 olefin of $\mathbf{2 3}$ completed the synthesis of 5-deoxyterreulactone $\mathrm{C}$ (28). f. Chemical structures of reagents used in the transformations shown in this figure. NADP, nicotinamide adenine dinucleotide phosphate; TMSCl, trimethylsilyl chloride; imid., imidazole; KHMDS, potassium bis(trimethylsilyl)amide; dpm, 2,2,6,6-tetramethyl-3,5heptanedionato; TBHP, tert-butyl hydrogen peroxide. 
a

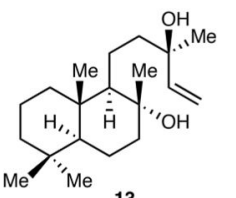

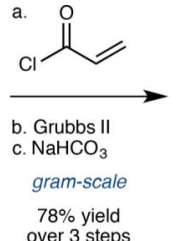

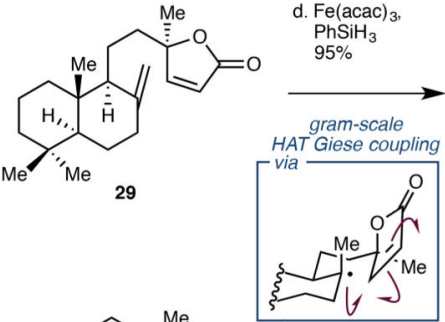

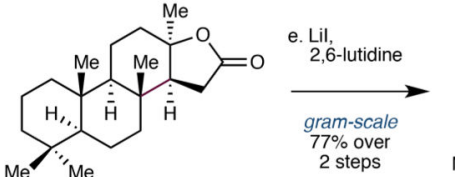

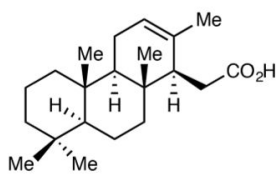
single g. $\begin{aligned} & \text { MERO1 } \\ & \text { L75A }\end{aligned} \underbrace{31} \begin{aligned} & \text { gram-scale } \\ & \text { hyyymatroxyliation } \\ & 62 \%\end{aligned}$

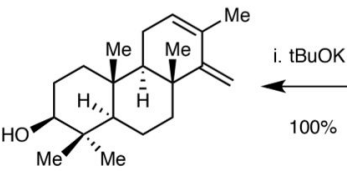
35
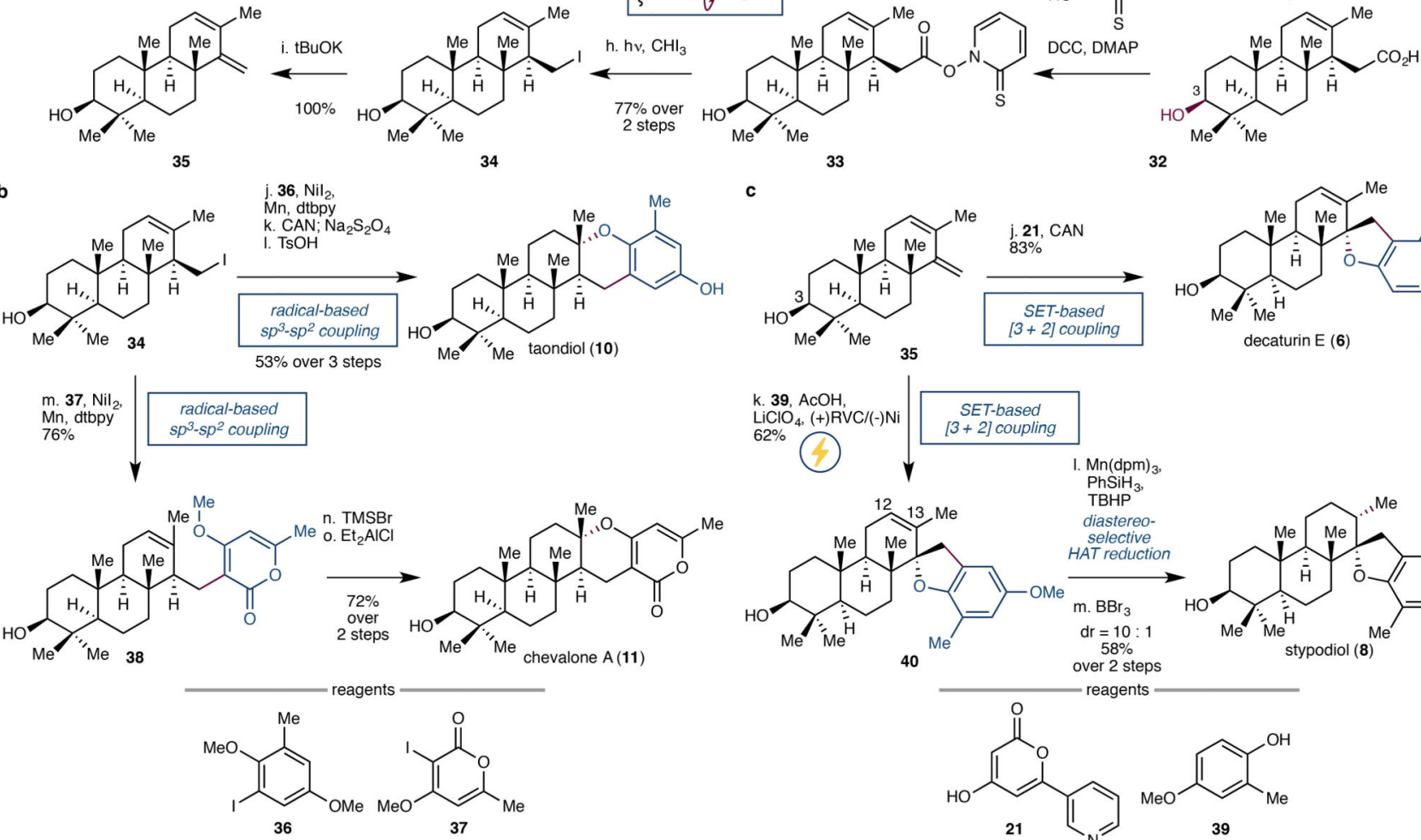

33

32
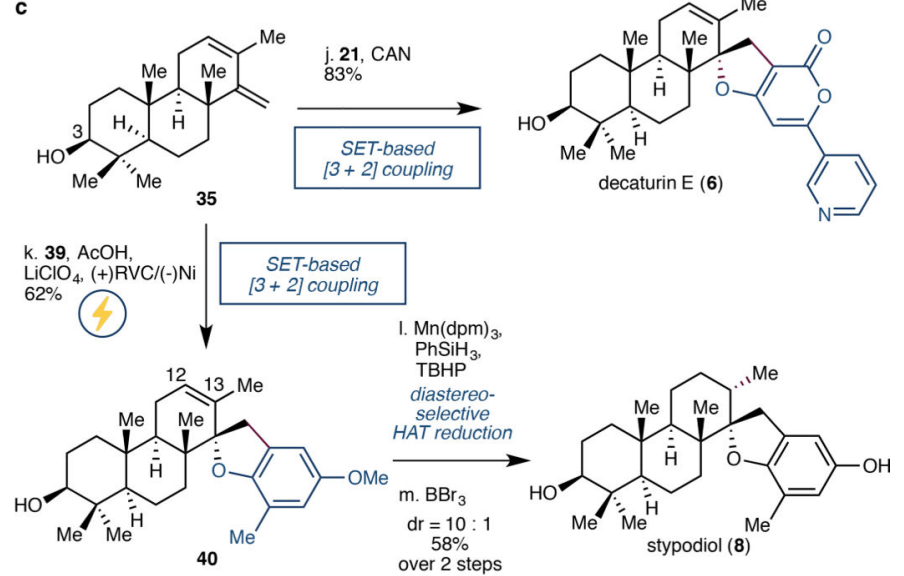

10 and $11: \cdot 11$ steps each, enantioselective

modular access from late-stage intermediate 3

- 2 key $\mathrm{C}-\mathrm{C}$ bonds, 2 key stereocenters set by radical-based logic

6 and 8: $\cdot 10$ and 12 steps, enantioselective

- modular access from late-stage intermediate 35

- 3 key $\mathrm{C}-\mathrm{C} / \mathrm{C}-\mathrm{O}$ bonds, 2 quaternary centers set by radical-based logic

Figure 4. Modular chemoenzymatic synthesis of diterpenic meroterpenoids.

a. Building blocks 34 and $\mathbf{3 5}$ were synthesised from sclareol (13) in 8 and 9 steps, respectively. Key transformations in the sequence include the use of HAT-based intramolecular Giese coupling to form the C-ring with complete diastereoselectivity and the use of biocatalytic hydroxylation with an engineered $\mathrm{P}^{450} 0_{\mathrm{BM} 3}$ to selectively install the $\mathrm{C} 3$ alcohol. b. Taondiol (10) and chevalone A (11) were synthesised in modular fashion from late-stage intermediate $\mathbf{3 4}$ via Ni-catalysed cross coupling with aryl iodides $\mathbf{3 6}$ and $\mathbf{3 7}$, respectively. Both 10 and 11 are accessed enantioselectively in 11 steps from 13; the full route involves biocatalytic installation of the key $\mathrm{C} 3-\mathrm{OH}$ and the formation of 2 key $\mathrm{C}-\mathrm{C}$ bonds and 2 key stereocentres using radical-based logic. c. Decaturin E (6) and stypodiol (8) were synthesised in modular fashion from late-stage intermediate 35 via SET-based [3 + 2] coupling with pyrone 21 and phenol 39, respectively. Enantioselective formation of $\mathbf{6}$ and $\mathbf{8}$ occurs in 10 and 12 steps, respectively, from 13. Key transformations include the biocatalytic installation of $\mathrm{C} 3-\mathrm{OH}$ and the formation of $3 \mathrm{C}-\mathrm{C} / \mathrm{C}-\mathrm{O}$ bonds and 2 quaternary centres using radical-based logic. Acac, acetyl acetone; DCC, N,N'- 
dicyclohexylcarbodiimide; DMAP, 4-dimethylaminopyridine; dtbpy, 4,4'-di-tert-butyl-2,2'dipyridyl; CAN, ceric ammonium nitrate; SET, single electron transfer; TMSBr, trimethylsilyl bromide; RVC, reticulated vitreous carbon. 